\title{
Magnitude and Pattern of Associated Complications of Bladder Outlet Obstruction Secondary to Benign Prostatic Hyperplasia at Yekatit 12 Hospital, Ethiopia: A Retrospective Descriptive Study
}

Zelalem Asefa ( $\square$ zelalem.asefa@aau.edu.et) Addis Ababa University

\section{Research Article}

Keywords: Benign prostatic hyperplasia, Bladder outlet obstruction, Associated complications, Ethiopia Posted Date: July 15th, 2021

DOI: https://doi.org/10.21203/rs.3.rs-662070/v1

License: (c) (i) This work is licensed under a Creative Commons Attribution 4.0 International License. Read Full License 


\section{Abstract}

Background: Benign prostatic hyperplasia (BPH) is the commonest cause of bladder outlet obstruction (BOO) in elderly males. It is characterised by lower urinary tract symptoms (LUTS) that include voiding/obstructive and storage/irritative symptoms. If untreated, patients could suffer from different associated complications such as hydronephrosis, urinary tract infection and even renal failure. Little is known about the magnitude and pattern of associated complications of BOO secondary to BPH.

Objectives: To assess the clinical profile, magnitude and pattern of associated complications of BOO among patients with BPH at Yekatit 12 hospital, Addis Ababa, Ethiopia.

Methods: A retrospective crossectional hospital based analysis of 225 patients who underwent prostatectomy for BOO secondary to BPH over a five year period, from January 1, 2015 to December 31, 2019 was done. Data were analysed using SPSS version 20.

Results: Frequency of micturition, dysuria and nocturia were the commonest presenting symptoms observed in $94.2 \%, 89.3 \%$ and $84.9 \%$ of the patients, respectively. One hundred and thirty $(57.8 \%)$ patients presented to the hospital 2 years after the onset of their symptoms. History of prehospital antibiotic treatment for recurrent urinary tract infection (UTI) was found in $64.4 \%$ of the patients. Associated complications of $\mathrm{BOO}$ secondary to BPH were observed in $44 \%$ of the patients. Acute urinary retention (AUR) and urinary tract infection (UTI) were the commonest associated complications observed in $50.7 \%$ and $30.7 \%$ of the patients, respectively. Hydronephrosis (21.8\%), renal impairment (19.1\%), urinary tract stones $(13.3 \%)$ and frank haematuria (4.4\%) were other complications observed in this series.

Conclusion: Storage/irritative LUTS predominate among our patients with BPH. Associated complications of $\mathrm{BOO}$ secondary to $\mathrm{BPH}$ were prevalent in the study. Acute urinary retention (AUR) and urinary tract infection (UTI) were the commonest associated complications observed. In order to avoid late diagnosis and reduce the occurrence of possible complications, clinicians should be aware of the possibility of BPH even if the storage/ irritative symptoms predominate in elderly males.

\section{Background}

Benign prostatic hyperplasia (BPH) is a pathologic process common in aging men. It is responsible for bladder outlet obstruction (BOO). It is characterised by lower urinary tract symptoms (LUTS) (1-3). Lower urinary tract symptoms include voiding or obstructive symptoms such as hesitancy, poor and/or intermittent stream, straining, feeling of incomplete bladder emptying, dribbling, etc, and storage or irritative symptoms such as frequency, urgency, urge incontinence, and nocturia. Lower urinary tract symptoms are nonspecific and may occur secondary to a wide variety of conditions (4-7). In older men, LUTS are commonly caused by BPH. Benign prostatic hyperplasia is primarily obstructive. If left untreated, its progressive LUTS may lead to associated complications such as recurrent urinary tract infection, stone formation, acute urinary retention, hydronephrosis and even renal failure $(3,8,9)$. These 
complications are rare in clinical trials. In real life practice, however, admissions with urinary retention, frank haematuria, recurrent urinary tract infection (UTI) and kidney injury are a regular event (9).

Little is known about the magnitude and pattern of associated complications of BOO secondary to BPH. The objectives of this study, therefore, were to assess the clinical profile, magnitude and pattern of complications of BOO secondary to BPH among patients who underwent prostatectomy at Yekatit 12 hospital $(\mathrm{Y} 12 \mathrm{H})$ with the hope that the information generated may serve as a base for further studies.

\section{Methods}

The study was conducted in the surgical department, general surgery unit of Yekatit 12 hospital (Y12H). The hospital is a teaching and referral hospital located in Addis Ababa, Ethiopia. It delivers general surgical services to elective and emergency patients.

A retrospective cross sectional hospital based study was applied. The sources of information were the operation theatre registration books and individual patients' medical records. All patients who were operated for major elective general surgical diseases from January 1, 2015 to December 31, 2019 were the source population. All patients who underwent elective prostatectomy for $\mathrm{BOO} 2^{\circ}$ to $\mathrm{BPH}$ during the study period whose medical records contained complete information of the required items were included in the study while patients whose charts were incomplete or lost were excluded from the study.

Data was collected using pretested and structured questionnaires. The structured questionnaire contained variables that included patients' age, clinical presentations, durations of symptoms, comorbid medical conditions, urinalysis, renal function test and abdominopelvic ultrasound results, associated complications, indications for preoperative catheterisation, postoperative complications, hospital stay and outcome of prostatectomy. The collected data was checked for consistency after which it was coded and fed into a computer. Data analysis was done using SPSS version 20.0 statistical window package and the results were presented in absolute numbers and percentages using frequency distribution tables and figures. Association test was carried out when necessary using chi square test and $p<0.05$ was considered statistically significant.

\section{Operational Definitions}

Associated complications of BPH refer to the possible complications that could occur if BOO due to BPH is untreated and range from UTI and stones in the urinary tract to renal damage and failure.

The diagnosis of $\mathrm{BOO} 2^{\circ}$ to $\mathrm{BPH}$ was made when digital rectal examination revealed an enlarged prostate of benign features in patients who presented with LUTS.

Information about the volume of the prostate, presence of hydronephrosis and urinary bladder stones was collected from the ultrasound report. 
Urinary tract infection was considered when urinalysis showed more than 5 white blood cells (WBCs) per high power field (HPF).

Renal function impairment was entertained when serum blood urea nitrogen (BUN) and creatinine levels were found elevated from the reference values.

\section{Results}

A total of 4260 patients underwent elective surgery for general surgical diseases during the study period. Out of these, 258 patients have undergone prostatectomy for $\mathrm{BOO} 2^{\circ}$ to $\mathrm{BPH}$ accounting for $6.1 \%$ of the total elective operations. Out of the 258 patients, medical records of 225 patients were possible to retrieve for analysis accounting for a retrieval rate of $87.2 \%$. The remaining records contained incomplete information or could not be traced and hence excluded from the study. The mean and median ages were $64.8 \pm 10.05$ and 65 years, respectively (Range 40-92 years). The majority of patients were in age group 60-69 years. Figure 1 shows the age distribution.

The clinical presentation of patients was shown on Table 1.

Table 1: Clinical Presentation of Patients with B00 $2^{\circ}$ to BPH, Y12H, January 1, 2015 - December 31 , $2019(N=225)$

\begin{tabular}{lc}
\hline \multicolumn{1}{c}{ Symptoms* } & Frequency (\%) \\
\hline Frequency of micturition & $212(94.2)$ \\
Dysuria & $201(89.3)$ \\
Nocturia & $191(84.9)$ \\
Urgency & $185(82.2)$ \\
Difficulty in passing urine & $179(79.6)$ \\
Poor urinary stream & $173(76.9)$ \\
Post micturition dribbling & $166(73.8)$ \\
Hesitancy & $143(63.6)$ \\
Sensation of incomplete voiding & $136(60.4)$ \\
Straining to urinate & $135(60.0)$ \\
Acute urinary retention & $114(50.7)$ \\
Intermittency & $91(40.4)$ \\
Haematuria & $30(13.3)$ \\
\hline
\end{tabular}

*Multiple symptoms were possible 
Frequency of micturition, dysuria and nocturia were the commonest presenting symptoms observed in $94.2 \%, 89.3 \%$, and $84.9 \%$ of the patients, respectively. The mean and median durations of symptoms at presentation were $21.8 \pm 15.69$ and 24 months, respectively (Range 1-120 months). One hundred and thirty $(57.8 \%)$ patients presented to the hospital 2 years after the onset of their symptoms. History of prehospital antibiotic treatment for recurrent urinary tract infection (UTI) was found in $64.4 \%$ of the patients. Among the patients with history of prehospital antibiotic treatment for UTI ( $N=145), 126$ (86.9\%) patients presented to the hospital 2 years after the onset of their symptoms and $19(13.1 \%)$ patients presented within 2 years of their symptoms $(P<0.0001)$. Overall, associated complications of $\mathrm{BOO} 2^{\circ}$ to BPH were observed in $44 \%$ of the patients (Table 2 ).

Table 2: Pattern and Frequency of Associated Complications of BOO $2^{\circ}$ to $\mathrm{BPH}, \mathrm{Y} 12 \mathrm{H}$, January 1, 2015 December 31, $2019(\mathrm{~N}=\mathbf{2 2 5})$

\begin{tabular}{lc}
\hline \multicolumn{1}{c}{ Symptoms* } & Frequency (\%) \\
\hline Frequency of micturition & $212(94.2)$ \\
Dysuria & $201(89.3)$ \\
Nocturia & $191(84.9)$ \\
Urgency & $185(82.2)$ \\
Difficulty in passing urine & $179(79.6)$ \\
Poor urinary stream & $173(76.9)$ \\
Post micturition dribbling & $166(73.8)$ \\
Hesitancy & $143(63.6)$ \\
Sensation of incomplete voiding & $136(60.4)$ \\
Straining to urinate & $135(60.0)$ \\
Acute urinary retention & $114(50.7)$ \\
Intermittency & $91(40.4)$ \\
Haematuria & $30(13.3)$ \\
\hline
\end{tabular}

* Multiple complications in a single patient were possible

** Urinary tract infection

Acute urinary retention (AUR) and urinary tract infection (UTI) were the commonest associated complications observed in $50.7 \%$ and $30.7 \%$ of the patients, respectively.

Hypertension, diabetes mellitus and bronchial asthma were comorbid medical conditions observed in $24 \%, 19.1 \%$ and $9.8 \%$ of the patients, respectively. One hundred thirty six $(60.4 \%)$ patients had indwelling catheter before surgery. Acute urinary retention (50.7\%), renal function impairment $(19.1 \%)$ and bleeding 
prostates $(4.4 \%)$ were indications for preoperative catheterisations. All patients had features of benign prostatic enlargement on digital rectal examination and ultrasound study. The mean and median volumes of the prostate were $80.7 \pm 39.33$ and $69 \mathrm{ml}$, respectively with a range of $26.5-234 \mathrm{ml}$.

Prostatectomy was done under spinal anaesthesia in 197 (87.6\%) patients and general anaesthesia was used in the rest. Figure 2 shows the postoperative complications.

The overall postoperative complication rate was $24.9 \%$. Urinary tract and surgical site infections were the commonest postoperative complications encountered in $14.7 \%$ and $13.8 \%$ of the patients, respectively. Postoperatively, patients were followed for the average and median durations of $7.5 \pm 4.91$ and 6 months, respectively with a range of $1-25$ months. Two patients died accounting for a mortality rate of $0.9 \%$.

\section{Discussion}

Benign prostatic hyperplasia (BPH) is the commonest cause of bladder outlet obstruction (BOO) in aging men. If untreated, patients could suffer from different associated complications such as urinary tract infection, hydronephrosis and renal failure $(3,8,9)$. This study attempted to assess the clinical profile, magnitude and pattern of associated complications of $\mathrm{BOO}$ among patients with $\mathrm{BPH}$. The mean age of patients was 64.8 years in this series and this was comparable to other studies $(10,11)$. Mean age of 70 years was reported by Botto et al (12). The lowest age with BOO due to BPH in Ugwumba et al (11), Botto et al (12), and Ibrahim et al (13) studies were 47, 49 and 50 years, respectively. It is observed that many men over the age of 40 will develop histologic benign prostatic hyperplasia, but not all will develop bothersome lower urinary tract obstruction (14). The lowest age in this series was 40 years. The majority of patients in this, Ugwumba et al (11) and Seife et al (15) studies were in age group 60-69 years. Most patients in Ibrahim et al series were between $70-79$ years (13). Benign prostatic hyperplasia is primarily obstructive and obstructive symptoms of LUTS are expected to predominate in patients' presentation. However, men with BPH more commonly present with irritative than obstructive symptoms (3) and this was in agreement to our study where most patients presented mainly with frequency of urination, dysuria and nocturia. Ibrahim et al (13) and Ahmed (16) also showed frequency of urination in $87 \%$ and $90.5 \%$ of their patients, respectively. About $50.7 \%$ of our patients had acute urinary retention and this was observed in $81.3 \%, 35.6 \%, 40 \%$ and $79 \%$ of the patients in Berhanu (10), Ugwumba et al (11), Seife et al (15) and Ahmed (16)) studies, respectively. The majority of patients in the Western world present apparently early with mildly enlarged prostates but in the developing countries most patients present very late with big prostates $(11,13)$. In this and Ibrahim et al studies $(13)$, the mean duration of symptoms at presentation were 21.8 and 25.8 months, respectively. The duration of symptom presentation ranged 1- 120 months in our series and this ranged 1-168 months in Ibrahim et al study (13). The mean volume of the prostate in this series was $80.7 \mathrm{ml}$. Mean prostatic volumes of $73.6 \mathrm{ml}(10), 100.7 \mathrm{ml}(11), 103.6 \mathrm{ml}$ (13) and $70 \mathrm{ml}$ (16) were reported in other studies. Deori et al reported mean prostatic volume of $43 \mathrm{ml}$ in their series (17).

Berhanu reported recurrent urinary tract infection (UTI) in $60.9 \%$ of his patients (10). History of prehospital antibiotic treatment for recurrent UTI was found in the majority of our patients and most of 
these patients presented to the hospital 2 years after the onset of their symptoms. Patients with $\mathrm{BOO} 2^{\circ}$ to BPH could have recurrent UTI and treatment with antibiotics would provide transient relief of symptoms until another episode of UTI with irritative symptoms occur for patients to revisit health institutions. Prehospital treatment for recurrent UTI and the transient relief of symptoms might be the reasons for some patients' remarkable late presentation to our hospital. Irritative symptoms of LUTS might mislead clinicians to delay the diagnosis and treatment of the primary cause unless careful history taking and physical examination including digital rectal examination are done and wide ranges of possible causes including BPH are considered in male patients after the age of 40 years (3-7).

Recurrent urinary retention was the predominant complication observed in the developed world (18), whereas features of late presentation such as urinary bladder diverticuli, stones, and impaired renal function were common in the developing countries $(19,20)$. Acute urinary retention, urinary tract infection and hydronephrosis were the principal associated complications observed in our study and the commonest associated complications observed in Ibrahim's et al series were urinary tract stones, groin hernia and impaired renal function (13). Our patients with acute urinary retention were managed with transurethral catheterisation and urinary tract infections were treated with antibiotics before surgery. Urinary bladder stones were removed simultaneously at the time of prostatectomy. Preoperatively, continuous transurethral catheter drainage was used to optimise the conditions of patients with bladder outlet obstruction due to BPH complicated by renal function impairment. Patients with frank haematuria were managed by continuous transurethral catheter irrigation and blood transfusion was given for those indicated before surgery.

Comorbid medical conditions are common among elderly males. Hypertension and diabetes mellitus were the main comorbid medical conditions observed in this study and similar findings were noted in other studies $(11,13)$.

Urinary tract and surgical site infections were the main postoperative complications observed in our patients. Bladder calculi and clot retention were the commonest complications reported by Ugwumba et al (11) and Ibrahim et al observed retrograde ejaculation, clot retention and wound infection as the main postoperative complications in their series (13). Wound infection, transient urinary incontinence and suprapubic leak were the commonest postoperative complications noted by Seife et al (15). Absence of some postoperative complications such as retrograde ejaculation and sexual dysfunction in this study may be due to the cultural barrier of providing such information. The mortality rate observed in this report was comparable to most African studies $(11,13)$ except to that of Berhanu where he reported no death in his study (10).

There are multiple limitations to this study. It was a hospital based, single centre study and the conclusions made might not represent the society at large. As it was a retrospective study, further clinical symptoms and associated complications of $\mathrm{BOO} 2^{\circ}$ to $\mathrm{BPH}$ could not be ascertained. Besides, some variables that could potentially contribute to associated complications such as the severity of bladder outlet obstruction were not directly assessed. 
In conclusion, irritative symptoms of LUTS were the predominant symptoms among our patients with $\mathrm{BOO}$ secondary to BPH. Transient relief of symptoms by antibiotic treatment might contribute to our patients' late presentation. Associated complications of $\mathrm{BOO} 2^{\circ}$ to $\mathrm{BPH}$ were prevalent in the study and acute urinary retention, urinary tract infection, hydronephrosis, renal function impairment, bladder stones and frank haematuria were the complications observed.

Clinicians should bear in mind the possibility of BPH as a cause and thoroughly examine and further investigate when male patients after the age of 40 years present with predominantly irritative LUTS. This would help to detect BPH early and reduce the occurrence of associated complications which may be associated with increased morbidity and mortality. Further studies are recommended.

\section{List Of Abbreviations}

$\mathrm{BPH}$ - benign prostatic hyperplasia

BOO - bladder outlet obstruction

AUR - acute urinary retention

UTI - urinary tract infection

LUTS - lower urinary tract symptoms

Y12H - Yekatit 12 hospital

SPSS - Statistical Package for the Social Sciences

WBC - white blood cell

HPF - high power field

BUN - blood urea nitrogen

\section{Declarations}

\section{Ethics approval and consent to participate}

Permission to conduct the study was obtained from Dr Misganaw Tesfa, head of the department of surgery and Mr Taye Zewdie, academic vice Provost of the hospital. Patients' names and other identifying characteristics were not documented to ensure confidentiality during data collection.

\section{Consent for publication}

Not applicable 
Availability of data and materials

The datasets used and/or analysed during the current study are available from the corresponding author on reasonable request

Competing interests

No

\section{Funding}

None

\section{Authors' contributions}

ZA analysed and interpreted the data. ZA read and approved the final manuscript.

\section{Acknowledgements}

I am very grateful to Dr Misganaw Tesfa, Mr Taye Zewdie, Dr Jatani Arero and W/o Senait Mekonnen for their valuable help.

\section{Author's information}

ZA graduated as a general surgeon in 2005. ZA is serving as a consultant surgeon and associate professor of surgery at Addis Ababa University, School of medicine, Addis Ababa, Ethiopia. ZA is involved in teaching general surgery for undergraduate medical students and postgraduate surgical residents. ZA is an academic coordinator and focal person for general surgery at university affiliated Yekatit 12 hospital.

\section{References}

1. Bosch JL., Hop WC, Niemer AO, Banqma CH., Kirkels WJ, Schroder FH. Parameters of prostate volume and shape in a community based population of men 55 to 74 years old, J. Urol Nov; 1994; 152(5 Pt 1):1501-1505.

2. Masumori N, Tsukamoto T, Kumamato Y, et al, Japanese men have smaller prostate volume but comparable urinary flow rates relative to American men; results of community based studies in 2 countries, J. Urol. 1996; Apr; 155(4):1324-1327.

3. Han M, Partin AW. Simple Prostatectomy. In: Alan J. Wein, Louis R. Kavoussi, Alan W. Partin, eds. Campbell-Walsh Urology, 11th edn. Philadelphia, Elsevier: 2016; Vol 3. 2535-2542

4. Herbert Lepor. Pathophysiology of Lower Urinary Tract Symptoms in the Aging Male Population. Rev Urol. 2005; 7 (Suppl 7): S3-S11 
5. Chapple CR, Wein AJ, Abrams P, et al.: Lower urinary tract symptoms revisited: a broader clinical perspective. Eur Urol. 2008; 54 (3): 563-9.

6. Arman A. Kahoker, Peter J. Gilling: Recent advances in the understanding of male LUTS [version 1; referees: 2 approved]. F1000Res. 2016; 5: Pii: F1000 Faculty Rev-715.10. 12688/f1000 research.8638.1

7. Lower Urinary Tract Symptoms- Differential Diagnosis and Management. https://teachmesurgery.com; Accessed 12 August 2020

8. Levi A Deters, Edward D Kim: Benign Prostatic Hyperplasia (BPH). http://emedicine.medscape.com; Accessed 21 March 2021

9. Mark J. Speakman, Xi Cheng. Management of the Complications of BPH/BOO. Indian J Urol. 2014 Apr- Jun; 30(2): 208-213

10. Doi: $10.4103 / 0970-1591.127856$

11. Berhanu N. A. "Safety and efficacy of transversical prostatectomy done at primary general hospital setting in Ethiopia," East and Central African Journal of Surgery, 2008; vol. 13, pp. 53-60.

12. Ugwumba FO, Ozoemena OF, Okoh AD, Echetabu KN, Mbadiwe OM. Transvesical Prostatectomy in the Management of Benign Prostatic Hyperplasia in a developing country. Nigerian Journal of Clinical Practice 2014; Volume 17, Issue 6, 797-801.

13. Botto H, Lebret T, Barre P, et al. Electrovaporization of the prostate with the Gyrus device. J Endourol; 2001; 15:313-6.

14. Ibrahim Ahmed Gadam, Ali Nuhu, Suleiman Aliyu. Ten- Year Experience with Open Prostatectomy in Maiduguri, Nigeria. ISRN Urology 2012.

15. Claus G. Roehrborn. Benign Prostatic Hyperplasia. In: Alan J. Wein, Louis R. Kavoussi, Alan W. Partin, eds. Campbell-Walsh Urology, 11th edn. Philadelphia, Elsevier: 2016; Vol 3. 2535-2542

16. Henok Seife, Andualem Deneke. A descriptive study of transvesical prostatectomy outcomes at a general hospital in Addis Ababa, Ethiopia. East and Central African Journal of Surgery Vol.23 No. 1 (2018)

17. DOI: $10.4314 /$ ecajs.v23il.5

18. Ahmed AA. Transvesical Prostatectomy in Tikur Anbessa Hospital. East Afr Med J. 1992 Jul; 69 (7): 378-80

19. Rupam Deori, Bijoyananda Das, Mustafa Abdur Rahman. A study of relationship of prostate volume, prostate specific antigen and age in benign prostatic hyperplasia. International Journal of Contemporary Medical Research 2017; 4(7): 1582-1586

20. Kurokawa K., Tamura Y., Ogura H. et al. "Open surgery of elderly patients with benign prostatic hypertrophy," Acta Urologica Japonica, 1990; vol. 36, no. 10, pp. 1167-1172.

21. Akpo E. E. and Akpo M.O., "Giant benign prostatic hyperplasia in a Nigeria: report of a case," The Internet Journal of Urology 2011; vol. 8, no. 1. 
22. Yonou H., Goya M., Miyazato M., Sugaya K., Hatano T., and Ogawa Y., "Giant prostatic hypertrophy: a case report," Hinyokika Kiyo, 1999; vol. 45, no. 5, pp. 375-377.

\section{Figures}

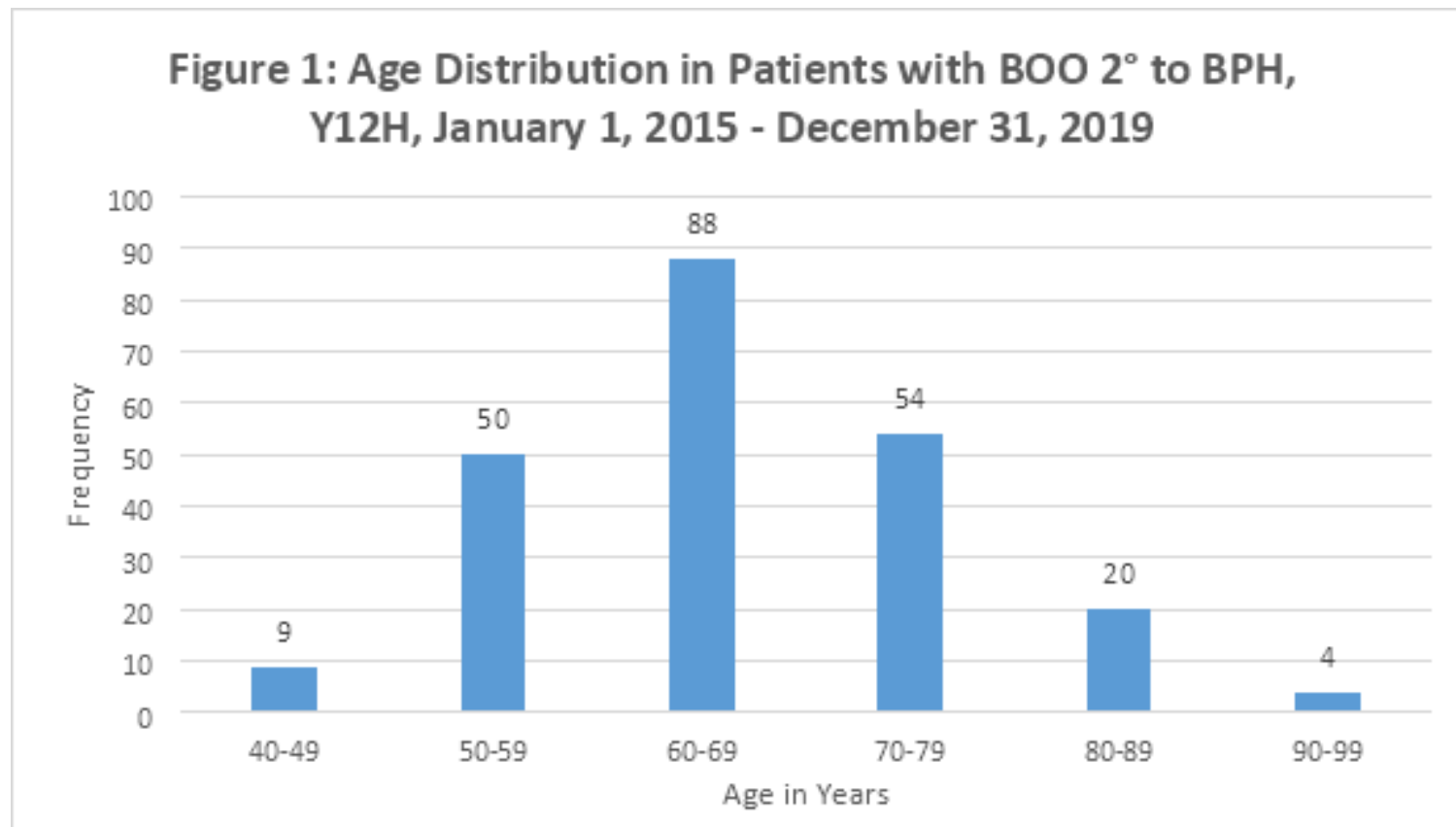

Figure 1

Age Distribution in Patients with BO0 $2^{\circ}$ to BPH, Y12H, January 1, 2015 - December 31, 2019

Figure 2: Postoperative Complications after

Prostatectomy in Patients with $\mathrm{BOO} 2^{\circ}$ to $\mathrm{BPH}, \mathrm{Y} 12 \mathrm{H}$,

January 1, 2015 - December 31, 2019

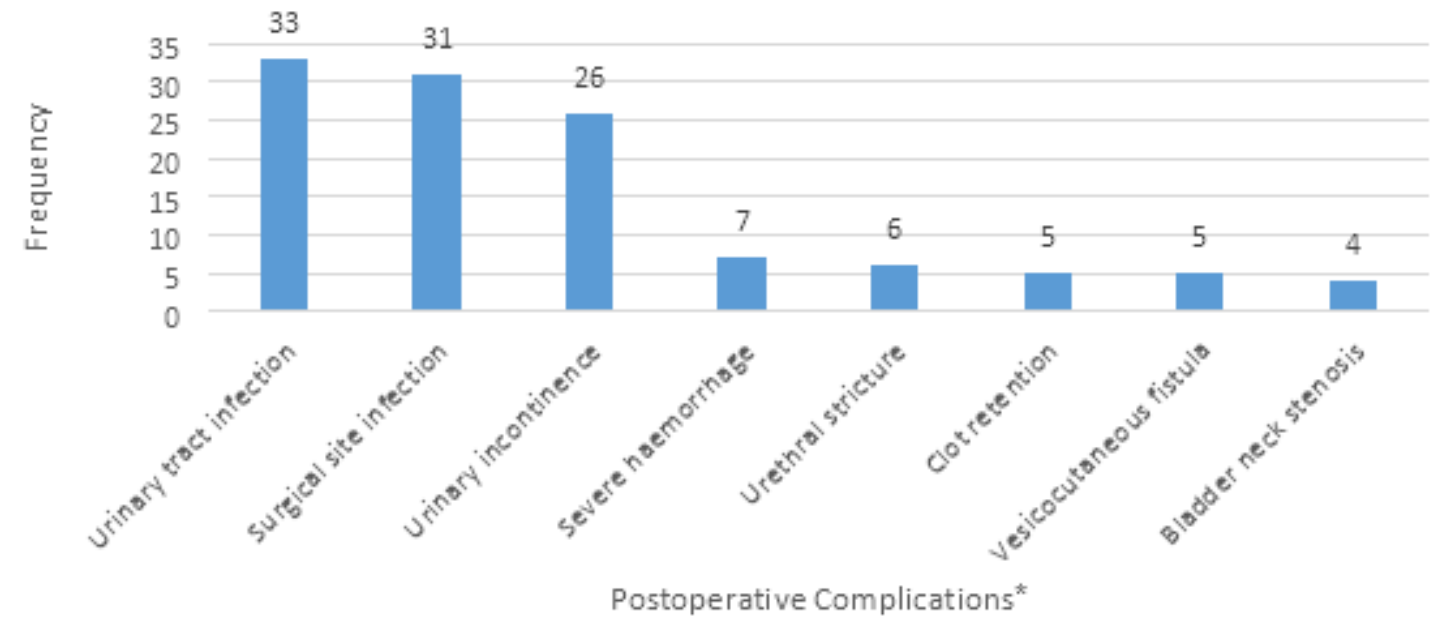

Figure 2 
Postoperative Complications after Prostatectomy in Patients with BOO $2^{\circ}$ to $\mathrm{BPH}, \mathrm{Y} 12 \mathrm{H}$, January 1, 2015 - December 31, 2019* Multiple complications were possible 